\title{
A review of clinical profile, complications and antibiotic susceptibility pattern of extensively drug-resistant (XDR) Salmonella Typhi isolates in children in Karachi
}

\author{
Saba Shahid ${ }^{1 *}$, Marvi Mahesar ${ }^{2}$, Nida Ghouri ${ }^{2}$ and Saba Noreen ${ }^{1}$
}

\begin{abstract}
Background: Enteric fever is a systemic infection caused by Salmonella enterica serovar Typhi and Salmonella enterica serovar Paratyphi $A, B$, and $C$. There is an emergence of Typhoid fever caused by extensively drug-resistant Salmonella Typhi strain called XDR S.Typhi. This strain is resistant to recommended first-line antibiotics and cephalosporins. WHO estimated 5274 cases of XDR S.Typhi in Karachi from November 2016 to December 2019. This study aims to determine clinical course, complications and response to treatment of XDR S.Typhi among the pediatric population coming to Indus Hospital.
\end{abstract}

Method: We reviewed the records of children who had culture-proven XDR S.Typhi infection at Indus Hospital from July 2017 to December 2018. A pre-designed data abstraction form was used to record information about seasonality, demographic details, clinical features and course, treatment, complications and outcomes of the cases of XDR S.Typhi.

Results: The records of 680 children were reviewed. The median (IQR) age of the patients was 5 (2-8) years. More than half $(n=391,57.5 \%)$ of the patients were males. The outcomes were recorded in 270 (40\%) patients. Out of these, 234 (86.7\%) children got cured within 14 days, while a delayed response to antibiotics was noted in 32 (11.9\%) children. Seventy-six (29\%) children recovered on a combination of meropenem and azithromycin, 72 (27\%) got cured on azithromycin alone, while 15 (6\%) responded to meropenem alone.

Conclusion: Our review indicated that children under 5 years of age were affected more with XDR S.Typhi. Azithromycin alone or in combination with meropenem were effective antibiotics for treating XDR S.Typhi in children.

Keywords: XDR S.Typhi, Seasonality, Children, Antibiotics

\section{Background}

Enteric fever is a systemic infection caused by Gramnegative bacteria, Salmonella enterica serovar Typhi, Salmonella enterica serovar Paratyphi $A, B$, and $C$ [1]. The disease is estimated to affect approximately 11-21 million individuals globally on an annual basis and has

*Correspondence: saba.shahid@tih.org.pk

${ }^{1}$ Department of Pediatrics, The Indus Hospital, Karachi, Pakistan

Full list of author information is available at the end of the article a high mortality rate [2]. Recent data showed that globally 200,000 deaths result annually due to enteric fever [3]. The burden of disease of enteric fever is the highest in Asia; $93 \%$ of the global cases are reported from within this region [3]. Southeast Asia has the third-highest incidence within the region, with approximately 110 cases/100,000 population. The estimated incidence of enteric fever in Pakistan was 413/100,000 in children aged 2-4 years and $573 / 100,000$ in children aged $5-15$ years [1]. 
Over time, Salmonella Typhi has developed resistance to many antibiotics, resulting in the emergence of multi-drug resistant Salmonella Typhi (MDR S.Typhi). These strains have shown resistance to first-line drugs, namely ampicillin, chloramphenicol and trimethoprimsulfamethoxazole. However, they are sensitive to thirdgeneration cephalosporins [4]. This strain of enteric fever has been endemic in countries like Pakistan, India, Nepal and Bangladesh since the 1980s [4]. However, a review of S.typhi and Paratyphi-A conducted in Pakistan from 2009 till 2011 showed an increased frequency of MDR S.Typhi (91.7\%) and two cases of S.Typhi resistant to cephalosporin [5].

In November 2016, a massive outbreak of ceftriaxoneresistant S.Typhi was reported among children residing in Hyderabad, Pakistan. Around 486 cases were reported, and consumption of contaminated drinking water was linked with the infection [5]. These strains were called extensively drug-resistant Salmonella Typhi (XDR S.Typhi) as they showed resistance to all the recommended antibiotics for typhoid fever, including thirdgeneration cephalosporin. In addition, the strains of XDR S.Typhi showed sensitivity to either carbapenem (meropenem and ertapenem) or azithromycin [6]. Since the outbreak of typhoid fever caused by XDR S.Typhi in Hyderabad, many other similar cases have been reported. WHO estimated 5274 cases of XDR S.Typhi in Karachi from November 2016 to December 2019 [7]. In Pakistan, cases of XDR S.Typhi are routinely managed with azithromycin and meropenem alone or in combination.

XDR S.Typhi is a new strain and may have unique clinical manifestations and outcomes compared to MDR S.typhi infection. Unfortunately, there is a scarcity of data on clinical features and response to treatment in children suffering from XDR S.Typhi nationally and in other parts of the world. Therefore this study is done to determine clinical course, complications and outcomes of XDR S.Typhi among the pediatric population coming to Indus Hospital. Primary endpoints included clinical manifestations, complications, response to treatment and outcome of the participants. Secondary endpoints included the seasonality of the infection and geographic distribution of the cases.

\section{Methods}

\section{Study design and data collection}

A retrospective chart review of blood culture-confirmed XDR S.Typhi cases was conducted at The Indus Hospital, Korangi campus from 1st July 2017 to 31st December 2018. The Indus Hospital is a free, multi-disciplinary tertiary care hospital situated in Karachi. Pediatric services include 115 beds. Pediatric services at Korangi campus include pediatric oncology, critical care and general ward.
Children with ages ranging from birth till 15 years were included in the study. Data from both inpatient and outpatient (OPD) records were obtained. Blood culture-confirmed cases of XDR S.Typhi, which showed resistance to the five classes of antibiotics (ampicillin, chlorampheni$\mathrm{col}$, trimethoprim-sulfamethoxazole, fluoroquinolones, and 3rd generation cephalosporin (ceftriaxone or cefixime), were included in the study. All children with XDR S.Typhi identified during the study period were included. Clinical features and lab investigations done at the time of presentation were noted for those children who left against medical advice (LAMA) or did not seek treatment or returned to hospital for follow-up visits. Outcomes were noted for those children who had a complete record. After discharge, admitted children were followed in OPD on day 14 to check the clinical response to treatment. The children were considered cured if fever defervescence was achieved within 14 days of starting antibiotics. For children who did not show fever defervescence on day 14, antibiotics were continued, and blood culture was repeated. These children were considered to have delayed response to antibiotics. The second follow-up was done for late responders on day 21. During the second follow up clinical response to antibiotics was noted, and blood culture was repeated if fever persisted. Children who missed their appointment were reminded through phone calls as a routine practice in the institute.

A pre-designed data abstraction form was used to record detailed information about the month-wise distribution of cases, patient demographics, signs and symptoms, clinical course, time in days to defervescence from infection, details of antimicrobial therapy, complications and outcomes. In addition, laboratory parameters were also recorded. These included complete blood count $(\mathrm{CBC}), \mathrm{C}$-reactive protein (CRP), liver function test (LFTs), serum electrolytes, creatinine, cerebrospinal fluid analysis, ultrasound and CT scan reports. Data of complications retrieved from the medical record included electrolyte imbalances, haematological complications like cytopenias, cholecystitis, hepatitis, ascites, pleural effusion, shock, renal dysfunction, neurological complications like fits, encephalitis, encephalopathy and aphasia.

Treatment options noted in our study reflected the treatment protocols of our institute. All children suspected of having enteric fever were empirically treated with cefixime or ceftriaxone, depending on disease severity. Upon confirmation of XDR S.Typhi based on blood culture, antibiotics were switched to either azithromycin or meropenem. Uncomplicated cases of XDR S.Typhi were given azithromycin for 10 days, while complicated cases were treated with meropenem for 14 days. Admitted children receiving meropenem were discharged on oral azithromycin to complete treatment of 14 days. 
Meropenem was given Intravenously (IV) at a dose of $20-40 \mathrm{mg} / \mathrm{kg}$ three times a day, while azithromycin was given orally at $20 \mathrm{mg} / \mathrm{kg} /$ day.

Three to five ml of venous blood sample was drawn and blood was inoculated into BacT/Alert culture bottles of $\mathrm{BD}$ company. Additional subcultures were performed if bacterial growth was suspected on visual identification. Subculture was done on Mac-Conkey agar and Blood agar plates. Non-lactose fermenting colonies isolated on Mac-Conkey agar were biochemically tested for Salmonella typhi and confirmed by Salmonella specific anti-sera. Biochemical tests done to identify Salmonella species included motility, indole, citrate utilization, triple-sugar iron and sulfide production test.

XDR S.Typhi was identified on basis of antibiotic sensitivity which was tested by Kirby-Bauer disc diffusion technique on Mueller Hinton agar with standard antimicrobial discs. Antibiotic susceptibility for ampicillin, chloramphenicol, trimethoprim-sulfamethoxazole, ciprofloxacin, cefixime, ceftriaxone, imipenem, ertapenem, meropenem and azithromycin was performed according to CLSI guidelines 2018 [8]. For quality control E. coli ATCC 25922 was used as a reference strain in the disk diffusion susceptibility test.

We obtained approval from the ethical review committee (ERC) of our institute. We were granted ERC exemption, as this study is a retrospective chart review.

\section{Case definitions}

Non-resistant typhoid fever:Typhoid fever caused by S. Typhi or S.Paratyphi A, B or C strains sensitive to firstline drugs (ampicillin, chloramphenicol and trimethoprim-sulfamethoxazole, quinolone) and third-generation cephalosporins (cefixime and ceftriaxone) [5]. Multi-drug resistant Salmonella Typhi (MDR S-Typhi): Typhoid fever caused by S.typhi and or S.paratyphi A, B or $\mathrm{C}$ strains which are resistant to first-line drugs (ampicillin, chloramphenicol, and trimethoprim-sulfamethoxazole) [5].

Extensively drug-resistant Salmonella Typhi (XDR S.Typhi):Typhoid fever caused by $S$.

typhi strains that are resistant to all the recommended antibiotics for typhoid fever, including ampicillin, chloramphenicol, trimethoprim-sulfamethoxazole, quinolones (nalidixic acid/ciprofloxacin) and third-generation cephalosporin( cefixime and ceftriaxone) [5].

Cured:Cured was defined as defervescence of fever within 14 days of starting antibiotics.

Delayed response:It was defined as either persistence of fever or positive blood culture beyond 14 days of treatment.
Treatment failure:It was defined as persistence of fever or positive blood culture beyond 21 days of starting antibiotics or relapse of disease within 30 days of completion of antibiotics.

Lost to follow up:It was defined as children who did not seek treatment or returned to the hospital for a followup visit after the initial visit.

Renal impairment:Increased creatinine $>1.5$ times upper limit of normal or decreased in urine output $<0.5 \mathrm{ml} / \mathrm{kg} / \mathrm{h}$ for $6 \mathrm{~h}$ [9].

Hepatitis:Deranged liver function tests with ALT more t han twice the reference value with or without hyperbilirubinemia, impaired coagulation and hypoglycemia.

Cholecystitis:Evidence of inflamed gall bladder sludge in gall bladder on ultrasound.

Haematological complications:A decrease in two cell counts from given lab references were considered bicytopenia. A decrease in three cell counts from given lab references were considered pancytopenia.

Encephalopathy:Changes in mental status, confusion or stupor with normal CSF findings.

Encephalitis:Changes in mental status, confusion or stu por or signs of meningeal irritation with abnormal CSF findings.

Diarrhoea:Presence of loose or watery stools three times or more per day [10].

\section{Statistical analysis}

Data were analysed using SPSS version 24. Descriptive statistics were run for all continuous variables, exploring skewness and kurtosis. Mean with standard deviation was reported for normal distribution, while median with interquartile range was reported for skewed distribution.

\section{Results}

A total of 1518 patients had blood culture positive for enteric fever during the study period. Out of these, 1341 patients were children, and 177 patients were adults.

Out of 1341 pediatric patients, 661 children were excluded from the final analysis as they had MDR typhoid or non-resistant typhoid infection. However, XDR S.Typhi infection was present in 680 children, and they were included in the final analysis (Fig. 1). More than half $(\mathrm{n}=391,58 \%)$ of the patients were males. The patients' median (IQR) age was 5 (IQR 2-8) years, with minimum and maximum 0-14 years. Out of these patients, 612 (90\%) children presented to the emergency department, followed by OPD 65(9.6\%). Around 101 (15\%) patients were admitted to the hospital, 98 (97\%) patients were admitted to the general ward, and the rest were admitted to the intensive care unit for treatment (Table 1). 
The majority of children, 540 (79\%), came from district East (Fig. 2). Two seasonal peaks were identified in the year 2018. One was in February-May 2018, and the second peak was observed in August-October 2018 (Fig. 3).

Out of 101 admitted patients, 69 children were cured within 14 days, while 32 patients had delayed response to treatment, as their blood culture was negative after 14 days. The average hospital stay of admitted children was 6 (IQR 3-9) days. The most frequent complications observed in admitted patients were mesenteric lymphadenopathy (18\%), encephalopathy (16\%) and hepatitis (14\%) (Table 1). Among the admitted patients, 4 cases of encephalitis were noted, out of which three children had aphasia on presentation. All children with encephalitis had full recovery without any residual weakness or speech difficulty. Mortality was seen in four admitted children. All the deceased children were less than 4 years of age and died within 3 days of admission except for one child who died after 14 days of admission. Among the children who died, two received meropenem, one child was given a combination of meropenem and azithromycin, and one child received a combination of ceftriaxone and meropenem (Table 2). A neonate was identified among the deceased children who acquired XDR S.Typhi through vertical transmission. Causes of death included multi-organ dysfunction, pulmonary haemorrhage, renal impairment and electrolyte imbalances.

Outcomes were recorded in $270(40 \%)$ patients; (Table 1). Out of these, 234 (86.7\%) children got cured, 196 children got cured based on fever defervescence, while 38 children had fever defervescence and culture clearance within 14 days. Delayed response to antibiotics beyond 14 days was noted in 32 (11.9\%) children (Table 2). However, all children with delayed response got fever defervescence by 21 days of starting antibiotics. Thus, we did not find any treatment failure in our study.

Out of 270 children whose outcomes were known, 76 (28\%) recovered on a combination of meropenem and azithromycin, 72 (27\%) got cured on azithromycin alone, while $15(6 \%)$ responded to meropenem alone. Eightyone $(30 \%)$ children got cured on other combinations, including ceftriaxone, carbapenem, and azithromycin (Table 2). Delayed response beyond 14 days was noted in $32(12 \%)$ children. Out of these, 24 children $(75 \%)$ were receiving various combinations of antibiotics, while seven $(21 \%)$ children received single antibiotic. Antimicrobial information was missing for one child (Table 2).

\section{Discussion}

Enteric fever caused by S.typhi continues to pose as a health burden globally, with the incidence being highest in low to middle-income countries (LMIC) due to poor infrastructure of public health [11]. According to the World Health Organization [12], Pakistan faced the largest outbreak of XDR S.Typhi in Hyderabad in November 2016, followed by a similar outbreak in Karachi. XDR S.Typhi is a novel strain that belongs to the H58 lineage. It has plasmid-encoded resistance and extended-spectrum $\beta$-lactamase (ESBL) gene responsible for resistance to both first and second-line antibiotics [13]. Klemm

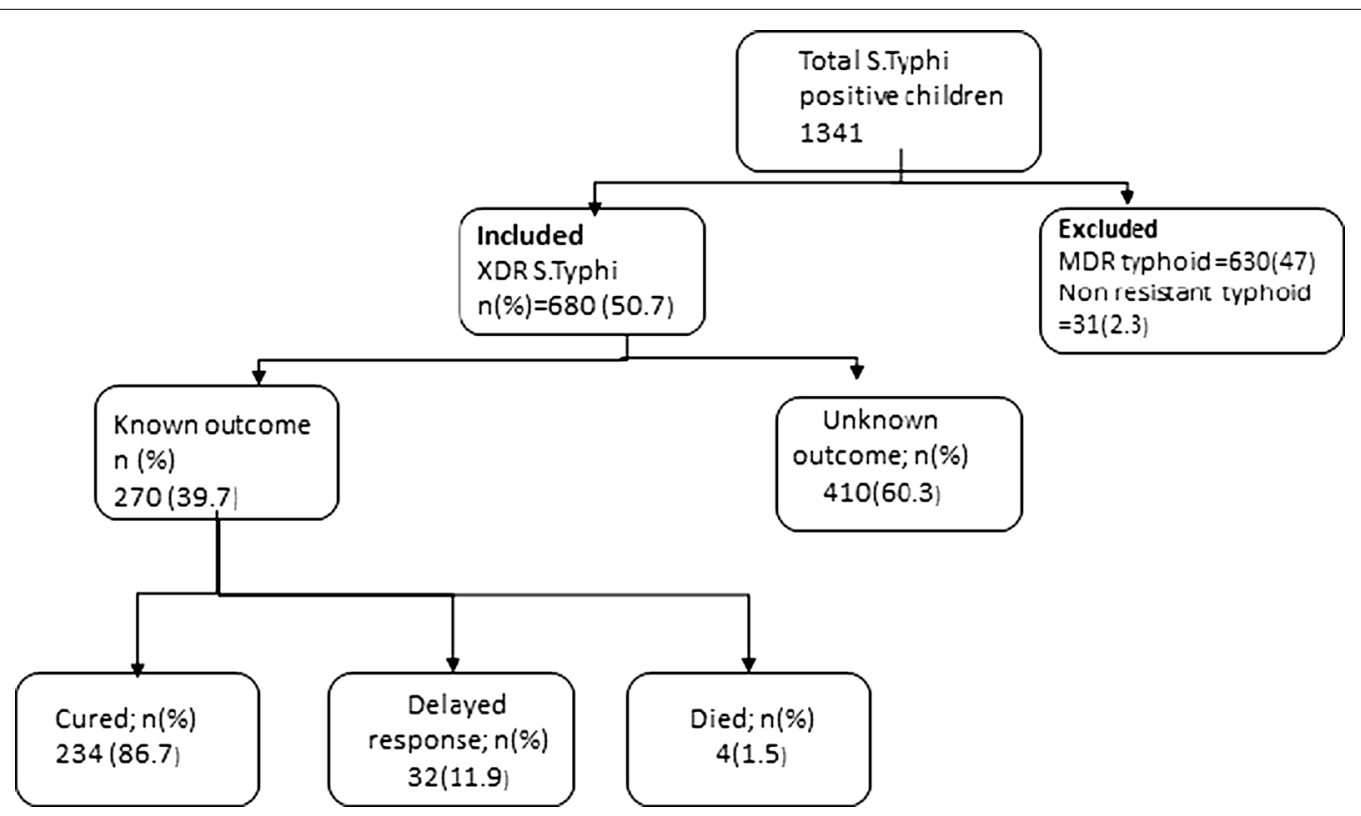

Fig. 1 Flow chart of patient inclusion, exclusion and outcomes 
Table 1 Demographic characteristics, clinical features, investigations, complications and outcome of study participants

Demographic features

Number (\%)

Male

Female

Age in years median (IQR)

Place of presentation

$E / R$

OPD

Inpatient

$3(0.4)$

Clinical features on presentation (680)

Fever

Vomitting

242 (36)

Diarrhea

174 (26)

Anorexia

$140(21)$

Cough

126 (19)

Abdominal pain

125 (18)

Hepatomegaly

$63(9)$

Splenomegaly

22 (3)

Urinary symptoms

$14(2)$

Rash

$12(2)$

Bleeding per rectum

4 (1)

Lab investigations Number (\%)

Raised CRP > 5 (415)

Hyponatremia (severe + moderate combined) (178)

Acidosis (178)

65 (37)

Hypokalemia (severe + moderate combined) (178)

59 (33)

Severe thrombocytopenia $\left(<50,000 \times 10^{9} / \mathrm{L}\right)(676)$

24 (4)

Raised serum creatinine (151)

$4(3)$

Severe anemia $(\mathrm{Hb}<5 \mathrm{gm} / \mathrm{dl})(676)$

Complications

Number (\%)

Mesentric lymphadenopathy (on abdominal ultrasound), (62)

Encephalopathy (101)

Hepatitis, LFT (81)

$11(14)$

Peritoneal free fluid (on abdominal ultrasound) (62)

$8(13)$

Bi-cytopenia (676)

$67(10)$

Pleural effusion (on abdominal ultrasound) (62)

$6(10)$

Cholecystitis (on abdominal ultrasound) (62)

$4(6)$

Shock (seen in 101 admitted patients)

$5(5)$

Encephalitis (seen in 101 admitted patients)

4 (4)

Aphasia (seen in 101 admitted patients)

Pancytopenia, done in 676

$16(2)$

Outcomes

Patients with known outcomes (270)

Cured

Delayed response

Died

Patients with unknown outcomes (410)

Lost to follow up

LAMA/referred out 


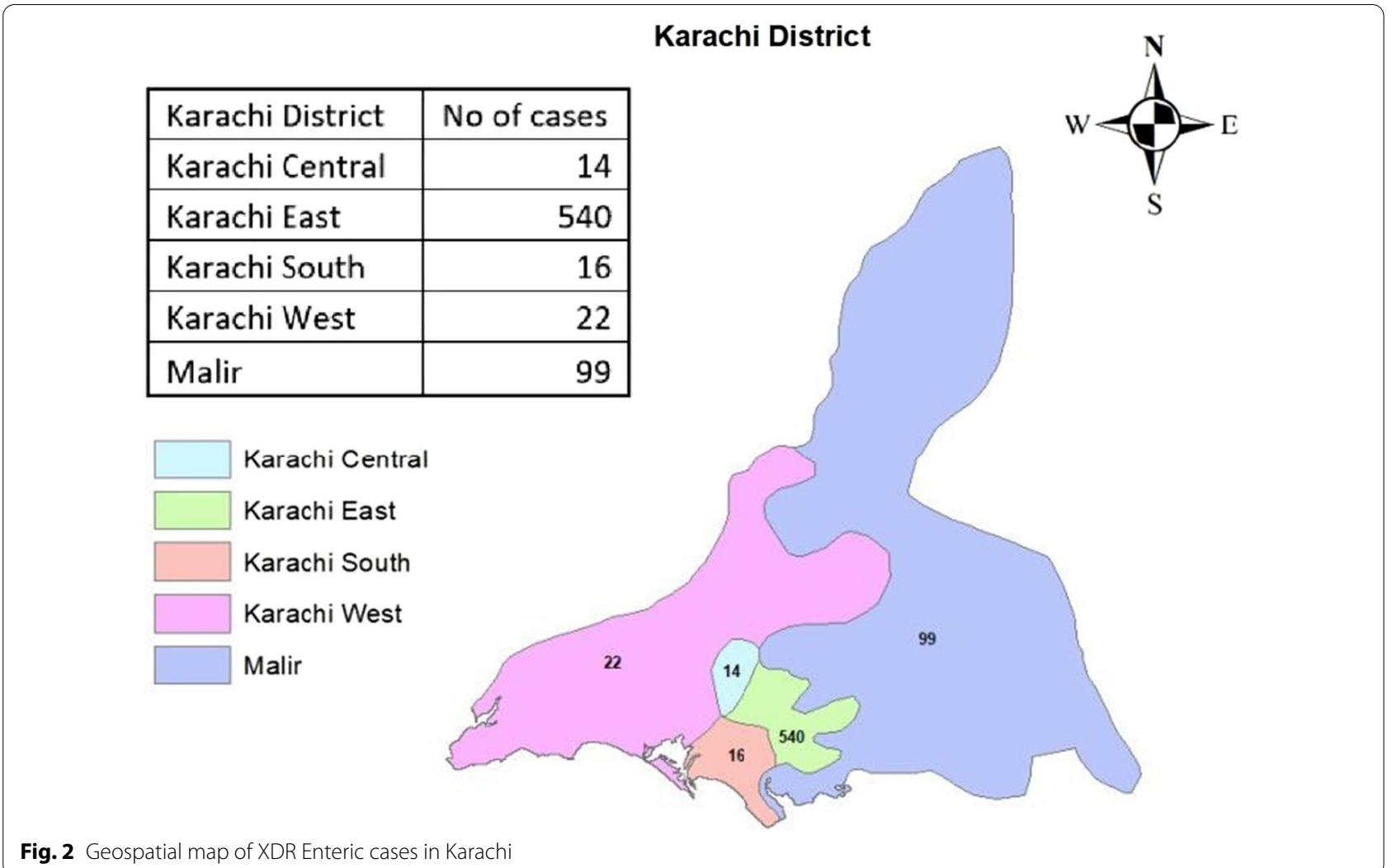

Fig. 2 Geospatial map of XDR Enteric cases in Karachi

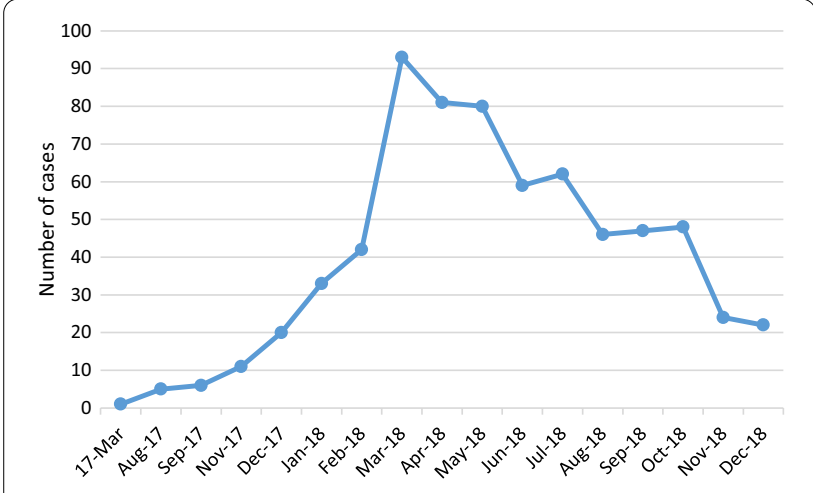

Fig. 3 Monthly distribution of XDR S.Typhi cases

et al. investigated the molecular epidemiology of XDR S.Typhi and found that these strains were also isolated from four different countries besides Pakistan, including India, Bangladesh, United Kingdom and Iraq. Of these, plasmid isolated from Iraqi strains were different while the rest of the strains were similar to Pakistan [13].

Out of 1518 total positive blood cultures for typhoid fever, 1341 (88\%) belonged to children. The median age of children with XDR S.Typhi was 5 (IQR 2-8) years. Literature from other parts of Pakistan has also reported a higher frequency of infection among children less than 5 years of age $[7,14]$. This could be explained by the fact that children have lower immunity and require lower bacterial doses to develop infection [15]. Most of the participants in our study belonged to the East district of Karachi, probably because Indus Hospital lies in the catchment area of this district. Many residential areas located in district East of Karachi comprise peri-urban slums, having unhygienic conditions, inadequate sewerage facilities and consumption of pipe-borne portable water supply by the people. The high burden of disease in these areas could be due to contaminated drinking water and mixing of drinking and sewage water, a finding which was also observed in Hyderabad [6].

Typhoid fever has been associated with considerable seasonal variations in different parts of the world [16]. In Pakistan, MDR typhoid fever peaks have been noted in May-June and in October. Both these periods are associated with monsoon rains in Pakistan. It has been postulated that seasonality is linked to increased consumption of contaminated local drinks and ice cream during the hot season and post-monsoon contamination of drinking water with rainwater [17]. We selected the year 2018 for our study because there was an outbreak of XDR S.Typhi in Karachi during this time. We observed two peaks in the number of cases, one in February-May 2018 and the second in August-October 2018. In addition, we 
Table 2 Clinical outcomes of patients on different antimicrobial therapy

\begin{tabular}{|c|c|c|c|c|}
\hline \multirow[t]{2}{*}{ Antimicrobial group $\mathrm{n}(\%)$} & \multicolumn{2}{|l|}{$\begin{array}{l}\text { Cured (266) } \\
\text { n (\%) }\end{array}$} & \multirow[t]{2}{*}{$\begin{array}{l}\text { Died (4) } \\
n(\%)\end{array}$} & \multirow{2}{*}{$\begin{array}{l}\text { Unknown } \\
\text { outcomes } \\
(410) \\
n(\%)\end{array}$} \\
\hline & $\begin{array}{l}\text { Response within } 14 \text { days } \\
\text { (234) }\end{array}$ & Response after 14 days (32) & & \\
\hline Azithromycin only & $69(29.5)$ & $3(9.4)$ & - & $41(10)$ \\
\hline $\begin{array}{l}\text { Combination of Azithromycin and Mero- } \\
\text { penem }\end{array}$ & $63(26.9)$ & $13(40.6)$ & $1(25)$ & $5(1.2)$ \\
\hline Meropenem only & $12(5.1)$ & $3(9.4)$ & $2(50)$ & $10(2.4)$ \\
\hline Cefixime & $3(1.3)$ & - & - & $26(6.3)$ \\
\hline Ceftriaxone & $3(1.3)$ & $1(3.1)$ & - & $74(18)$ \\
\hline Ciprofloxacin & $1(0.4)$ & - & - & \\
\hline Other combinations* & 70 (29.9) & $11(34.4)$ & $1(25)$ & $36(8.8)$ \\
\hline Not noted & $13(5.6)$ & $1(3.1)$ & - & $218(53.2)$ \\
\hline
\end{tabular}

*Other combinations included ceftriaxone and meropenem, ceftriaxone and azithromycin, imipenem and azithromycin

observed a peak number of cases from February to May 2018, which was a dry period in Karachi. This deviation from the usual seasonal pattern of the rainy season could be attributed to the XDR S.Typhi outbreak in many cities of Pakistan simultaneously in the year 2018. This finding is supported by another survey conducted in Lahore by Latif et al., who also reported peak cases of XDR S.Typhi from January to April 2018 [18]. There is a possibility that intercity travelling during these cities had contributed to the spreading of infection.

Electrolyte imbalance was one of the most common reasons for hospital admission and can be attributed to vomiting and diarrhoea seen in our patients. Hyponatremia was seen in $74(41 \%)$ children and hypokalemia in 59 (33\%) children. The hypovolemic shock was seen in 5 (5\%) children. All children with shock responded to fluids and inotropes except for one child who passed away. Neurological complications, which included encephalopathy and encephalitis, were seen in 20 children. Out of these, 15 children had seizures. Three children with fits had hyponatremia, four had encephalitis, and eight children had encephalopathy. Leung et al. [19] have reported 48 cases of enteric fever with encephalopathy. They found a strong correlation of encephalopathy with dehydration and low white blood cell count ( $\mathrm{p}$-value $<0.001)$. They postulated that heightened immune response in typhoid fever could lead to neurological complications. There is a possibility that children in our cohort also had an immunological mediated neuronal injury.

Four children developed aphasia, out of which three had encephalitis, and one had encephalopathy. All children recovered completely without any deficit. Few cases of aphasia caused by enteric fever have been reported [20, 21]. The literature reports multiple factors like electrolyte imbalance, cerebral injury and neurotoxins-associated injury in Broca's speech as the cause of motor aphasia in Enteric fever [20].

Mortality was seen in four children. Three children passed away within 3 days of admission, while the fourth child, a neonate, died in the 2nd week of infection. Delay in seeking medical treatment could be a contributing cause of early deaths in these children. Three hundred fifty-one children were lost to follow up in our study. The most probable explanation for this could be poverty, as most of our study participants belonged to low socioeconomic strata. These patients may find it challenging to afford travelling expenses.

In our study, 76 (29\%) children recovered on a combination of meropenem and azithromycin, 72 (27\%) got cured on azithromycin alone, while 15 (6\%) responded to meropenem alone.

Tayyaba et al. studied antibiotic susceptibility of XDR S.Typhi in Karachi and found equal susceptibility of XDR S.Typhi strains to both azithromycin (95\%) and meropenem (97\%) [22]. other studies also reported equal cure rates with both the drugs $[23,24]$. The number of cured children were almost similar in antimicrobial groups of azithromycin alone and azithromycin-meropenem combination, $76(28 \%)$ vs $72(27 \%)$. This finding has important future implications as our data suggests that XDR S.Typhi can be successfully treated with oral azithromycin, which will reduce the cost of treatment and hospital admission. Qureshi et al. [25] also reported that treatment of XDR S.Typhi by meropenem is more expensive than azithromycin. They reported an average daily cost of treatment for azithromycin to be US $\$ 5.87$ versus US $\$ 88$ for meropenem.

We found late responders but no treatment failure in our cohort. There is a possibility that children who had delayed response harboured XDR S.Typhi strains with 
a higher minimum inhibitory concentration (MIC) to azithromycin and meropenem. Since we did not perform MIC in our institute, we were unable to determine the association of MIC with clinical response.

Seven children got cured on cefixime, ceftriaxone and ciprofloxacin alone, although they were reported as resistant to those antibiotics. One explanation for this phenomenon could be that the resistant strains had higher MIC breakpoints and were missed by our lab due to lack of MIC. Another possibility could be that these patients also received azithromycin and did not communicate this information to us.

The strength of this study is that it is one of the few studies which examines the clinical course, outcomes, response to treatment and complications of XDR strain of S.Typhi amongst the pediatric population within Pakistan. However, the limitations of this study include the lack of use of MIC for culture and sensitivity, which would provide more information about response to antibiotics. Furthermore, more than half the children were lost to follow-up, and we could not determine the reasons for missed appointments. In addition, retrospective data had missing information on socio-demographics like drinking water quality, hygiene practices and the number of households.

\section{Conclusion}

This study confirms that XDR S.Typhi is common in children under 5 years of age. The use of azithromycin alone or in combination with meropenem for the treatment of XDR S.Typhi has shown promising results. However, the effectiveness of these regimens needs to be further investigated through randomised clinical trials. We advocate increasing nationwide awareness about the consumption of safe water, antibiotic stewardship and immunisation practices of children.

\begin{abstract}
Abbreviations
XDR: Extensively drug-resistant; MDR: Multi-drug resistance; WHO: World Health Organisation; IQR: Interquartile range; S.typhi: Salmonella Typhi; HMIS: Health Management Informatics System; CBC: Complete blood count; LFT: Liver function test; ALT: Alanine transaminase; CSF: Cerebrospinal fluid.
\end{abstract}

\section{Acknowledgements}

Not applicable.

\section{Authors' contributions}

SS and MM conceived the idea of the study and participated in study design and write up. SN and ND carried out data collection, MM and ND assisted with statistical analysis. All authors were involved in the coordination of the study, drafting the manuscript. All authors read and approved the final manuscript.

\section{Funding}

No funding was received for this research.

\section{Availability of data and materials}

The datasets used and analysed during the current study are available from the corresponding author on reasonable request.

\section{Declarations}

Ethics approval and consent to participate

Ethical approval was taken from the Institutional Review Board (IRB) of Interactive Research \& Development, registered with the U.S. Department of Health and Human Services, Office for Human Research Protections at The Indus Hospital. As per the IRB, written informed consent was not required from the patients involved in this study.

\section{Consent for publication}

Not applicable.

\section{Competing interests}

The authors declare that they have no competing interests.

\section{Author details}

${ }^{1}$ Department of Pediatrics, The Indus Hospital, Karachi, Pakistan. ${ }^{2}$ Indus Hospital Research Centre, The Indus Hospital, Karachi, Pakistan.

Received: 31 July 2020 Accepted: 23 August 2021

Published online: 03 September 2021

\section{References}

1. Mogasale V, Maskery B, Ochiai RL, Lee JS, Mogasale WV, Ramani E, et al. Burden of typhoid fever in low-income and middle-income countries: a systematic, literature-based update with risk-factor adjustment. Lancet Glob Health. 2014;2(10):e570-80.

2. Acosta CJ, Danovaro-Holliday MC, Baiqing D, Bhattacharya SK, Agtini MD, Bhutta ZA, et al. A study of typhoid fever in five Asian countries: disease burden and implications for controls. Bull World Health Organ. 2008;86:260.

3. Crump JA, Luby SP, Mintz ED. The global burden of typhoid fever. Bull World Health Organ. 2004;82:346-53.

4. Mandal S, DebMandal M, Pal NK. Antibiotic resistance of Salmonella enterica serovar Typhi in Kolkata, India, and in vitro experiments on effect of combined chemotherapy. Sci World J. 2012. https://doi.org/10.1100/ 2012/454059.

5. Akhtar S, Sarker MR, Jabeen K, Sattar A, Qamar A, Fasih N. Antimicrobial resistance in Salmonella enterica serovar typhi and paratyphi in South Asia-current status, issues and prospects. Crit Rev Microbiol. 2015;41(4):536-45.

6. Qamar FN, Yousafzai MT, Khalid M, Kazi AM, Lohana H, Karim S, et al. Outbreak investigation of ceftriaxone-resistant Salmonella enterica serotype Typhi and its risk factors among the general population in Hyderabad, Pakistan: a matched case-control study. Lancet Infect Dis. 2018;18(12):1368-76

7. Organization WH. Typhoid fever_Islamic Republic of Pakistan. Geneva: WHO; 2018

8. Wikler M. Methods for dilution antimicrobial susceptibility test $\mathrm{s}$ for bac teria that grow aerobically: approved standard, CLSI (NCCLS), 26 (2006) M7-A7. Citation: NK Soliman. 2019.

9. Sutherland SM, Byrnes JJ, Kothari M, Longhurst CA, Dutta S, Garcia P, et al. AKI in hospitalized children: comparing the pRIFLE, AKIN, and KDIGO definitions. Clin J Am Soc Nephrol. 2015;10(4):554-61.

10. Levine GA, Walson $J$, Atlas HE, Lamberti LM, Pavlinac PB. Defining pediatric diarrhea in low-resource settings. J Pediatr Infect Dis Soc. 2017;6(3):289-93

11. Radhakrishnan A, Als D, Mintz ED, Crump JA, Stanaway J, Breiman RF, et al. Introductory article on global burden and epidemiology of typhoid fever. Am J Trop Med Hyg. 2018;99(3 Suppl):4.

12. Organization WH. The work of WHO in the Eastern Mediterranean Region: annual report of the Regional Director 2019. 2020.

13. Klemm EJ, Shakoor S, Page AJ, Qamar FN, Judge K, Saeed D, et al. Emergence of an extensively drug-resistant Salmonella enterica serovar Typhi 
clone harboring a promiscuous plasmid encoding resistance to fluoroquinolones and thirdgeneration cephalosporins. mBio. 2018;9:e00105-18.

14. Saeed N, Usman M, Khan EA. An overview of extensively drug-resistant Salmonella Typhi from a tertiary care hospital in Pakistan. Cureus. 2019. https://doi.org/10.7759/cureus.5663.

15. Saha MR, Dutta P, Palit A, Dutta D, Bhattacharya MK, Mitra U, et al. A note on incidence of typhoid fever in diverse age groups in Kolkata, India. Jpn J Infect Dis. 2003;56(3):121-2.

16. Lin F, Vo AH, Phan VB, Nguyen T, Bryla D, Tran CT, et al. The epidemiology of typhoid fever in the Dong Thap Province, Mekong Delta region of Vietnam. Am J Trop Med Hyg. 2000;62(5):644-8.

17. Siddiqui FJ, Rabbani F, Hasan R, Nizami SQ, Bhutta ZA. Typhoid fever in children: some epidemiological considerations from Karachi, Pakistan. Int J Infect Dis. 2006;10(3):215-22

18. Latif S, Zia A, Ali SB, Hafeez S. Extensively drug resistant typhoid fever seen at Tertiary Care Hospital in Lahore. Inf Dis J Pak. 2019;28(3):51-4.

19. Leung DT, Bogetz J, Itoh M, Ganapathi L, Pietroni MA, Ryan ET, et al. Factors associated with encephalopathy in patients with Salmonella enterica serotype Typhi bacteremia presenting to a diarrheal hospital in Dhaka Bangladesh. Am J Trop Med Hyg. 2012;86(4):698.

20. Adnan M, Anjum A, Afroz S, Sardha M. Motor aphasia in the first week of enteric fever. S Afr J Child Health. 2012:6(1):26-7.
21. Adehossi E, Parola P, Brouqui P. Febrile Broca's aphasia: a rare presentation of typhoid fever. J Travel Med. 2003;10(3):192-3.

22. Anwar T, Rais H, Jamil MF, Safdar S, Amir MR, Altaf A, et al. Extended drug resistance in children with typhoid fever. Prof Med J. 2020;27(03):581-7.

23. Wong W, Al Rawahi H, Patel S, Yau Y, Eshaghi A, Zittermann S, et al. The first Canadian pediatric case of extensively drug-resistant Salmonella Typhi originating from an outbreak in Pakistan and its implication for empiric antimicrobial choices. IDCases. 2019;15:e00492.

24. Chatham-Stephens K, Medalla F, Hughes M, Appiah GD, Aubert RD, Caidi $\mathrm{H}$, et al. Emergence of extensively drug-resistant Salmonella Typhi infections among travelers to or from Pakistan-United States, 2016-2018. Morb Mortal Wkly Rep. 2019;68(1):11.

25. Qureshi S, Naveed AB, Yousafzai MT, Ahmad K, Ansari S, Lohana H, et al. Response of extensively drug resistant Salmonella Typhi to treatment with meropenem and azithromycin, in Pakistan. PLoS Negl Trop Dis. 2020;14(10):e0008682

\section{Publisher's Note}

Springer Nature remains neutral with regard to jurisdictional claims in published maps and institutional affiliations.
Ready to submit your research? Choose BMC and benefit from:

- fast, convenient online submission

- thorough peer review by experienced researchers in your field

- rapid publication on acceptance

- support for research data, including large and complex data types

- gold Open Access which fosters wider collaboration and increased citations

- maximum visibility for your research: over $100 \mathrm{M}$ website views per year

At BMC, research is always in progress.

Learn more biomedcentral.com/submissions 